Running head: ASIAN AMERICANS' SUICIDE IDEATION

\author{
Asian Americans' Family Cohesion and Suicide Ideation: \\ Moderating and Mediating Effects \\ Y. Joel Wong \\ Indiana University Bloomington \\ Soo Yun Uhm \\ University of Macau \\ Peiwei Li \\ Indiana University Bloomington
}

Accepted for publication on 9/12/2010

In press, American Journal of Orthopsychiatry

Date of draft: January 12, 2012 


\begin{abstract}
The purpose of this study was to examine the relationship between family cohesion and suicide ideation in a national, adult community sample of Asian Americans $(N=2072)$. The data for this study was drawn from the National Latino Asian American Study, the first national epidemiological study of Asian Americans' mental health. The results indicate that family cohesion was negatively related to suicide ideation. In addition, English proficiency moderated the relationship between family cohesion and suicide ideation. Family cohesion was more strongly related to suicide ideation among low English proficiency Asian Americans than among high English proficiency Asian Americans. Further, the findings are consistent with a model in which the relationship between family cohesion and suicide ideation was partially mediated by psychological distress. Practical implications of the results are discussed in terms of how mental health professionals can help strengthen family cohesion and prevent suicide ideation among Asian Americans.
\end{abstract}

Key words: Asian American, suicide, family cohesion, acculturation, English proficiency 


\section{Asian Americans' Family Cohesion and Suicide Ideation: \\ Moderating and Mediating Effects}

Suicidal behavior constitutes a major mental health risk in the United States. In 2005, 32,637 people died by suicide in the United States, representing an average of 89 suicides per day (American Association of Suicidology, 2008). There is a growing body of research documenting the risk and protective factors associated with suicidal behavior in the United States (Westefeld et al., 2000). However, this research has focused largely on European American samples rather than on racial and ethnic minority groups; specifically, there is a paucity of research on Asian Americans' suicidal behavior (Leong, Leach, Yeh, \& Chou, 2007). Although the rate of suicide deaths for Asian and Pacific Islander Americans (5.2 per 100,000) is less than the overall U.S. rate (Kung, Hoyert, Xu, \& Murphy, 2008), research on Asian Americans' suicidal behavior research remains critical; because factors associated with suicidal behavior cannot be divorced from one's cultural context, research findings based on European American samples may not necessarily generalize to Asian Americans (Choi, Rogers, \& Werth, 2009).

The purpose of this study, therefore, was to examine factors associated with suicide ideation in a national, adult community sample of Asian Americans. Although suicidal behaviors lie on a continuum, ranging from suicide ideation to attempted and completed suicides, we focused on suicide ideation in this study because it enables research on multiple correlates of suicidal behavior using large samples of live participants within a short period of time (Maris, Berman, \& Silverman, 2000). Moreover, suicide ideation is a robust predictor of suicide attempts and deaths (Mann, 2002) as well as a major public health problem in its own right because of its tremendous emotional and financial cost (Joiner, Van Orden, Witte, \& Rudd, 2009).

\section{Family Cohesion}


Recently, Joiner (2005) proposed an interpersonal theory of suicide that is based, in part, on the premise that suicide desire is engendered by the thwarting of one's basic interpersonal needs. Specifically, suicide desire develops as a result of perceived burdensomeness to others and failed belongingness, that is, feeling that one is alienated from a valued social group. Joiner et al. (2009) reviewed numerous studies showing that indicators of unmet interpersonal needs were associated with suicide outcomes among samples of largely European American adults. Although the interpersonal theory of suicide has yet to be applied empirically to Asian Americans, Author and ZZ (in press) proposed that its emphasis on unmet interpersonal needs is congruent with Asian cultural beliefs that suicidal behavior is primarily a response to socially stressful situations (Range et al., 1999; Shiang et al., 1997). In Asian cultures, one's well-being is viewed as more closely connected to the nature of interpersonal relationships than one's internal attributes (Markus \& Kitayama, 1991); accordingly, the thwarting of Asian Americans' interpersonal needs may be a particularly pernicious suicide risk.

In this study, we applied the construct of failed belongingness in Joiner's (2005) interpersonal theory to the study of family cohesion and suicide ideation. We focused on family cohesion as an indicator of belongingness to a valued social group because it is a core Asian cultural value - in many Asian cultures, there is an emphasis on filial piety, subjecting one's needs to those of the family and maintaining harmonious family relationships (Kim, Atkinson, \& Umemoto, 2001). Indeed, the central role of the family as a social support system for Asian Americans has been consistently observed across diverse Asian cultural groups (see Inman \& Yeh, 2007, for a summary). Because the family, rather than society, is viewed as one's primary source of security (Tsoh et al,. 2005), problems in family relationships may constitute a major threat to Asian Americans' sense of self and well-being. Moreover, the disclosure of personal 
problems to non-family members is frowned upon in many Asian cultures (Kim et al., 2001); therefore, a lack of family cohesion may result in many Asian Americans losing their main source of social support. Consequently, a lack of family cohesion may be a risk factor for suicidal behavior among Asian Americans (Leong et al., 2007). Consistent with these notions, studies have found that family conflicts and problems were related to suicidal behaviors among Asian Americans college students (Author, XX, \& YY, 2009) and adolescents (Lau, Jernewall, Zane, \& Myers, 2002).

\section{English Proficiency}

In addition to family cohesion, acculturation may be a culturally-relevant factor associated with Asian Americans' suicidal behavior (Leong et al., 2007). Defined as the process of adapting to the norms of the dominant European American culture (B. S. K. Kim, 2007), acculturation is particularly salient to the study of Asian Americans' mental health because immigrants comprise $69 \%$ of the Asian population in the United States (U.S. Census Bureau, 2000). The process of acculturation may be especially stressful for Asian American immigrants who might face challenges associated with language barriers, unfamiliarity with new cultural norms, and disruptions to family relationships (Hwang, Chun, Takeuchi, Myers, \& Siddart, 2005). Studies have shown that behavioral indicators of acculturation were negatively associated with suicidal behavior among Asian American college students (Jha, 2001) and adolescents (Lau et al., 2002). In the current study, we focused on one dimension of behavioral acculturation Asian Americans' English proficiency - because language may be the main means through which cultural information and meanings are conveyed among ethnic minorities (Kang, 2006). In addition, Lam, Pacala and Smith (1997) have argued that command of the English language is a central component of acculturation because it enables immigrants to work independently in 
American society. Lam et al. found that English proficiency was negatively associated with depression among elderly Chinese Americans. In another study, English proficiency was related to marital and family life satisfaction among Korean American women (Jeong \& Schumm, 1990). Finally, Kang found that English language competence, but not other behavior and identity-based dimensions of acculturation, was associated with indicators of psychological adjustment among Asian American college students. Nevertheless, no study has tested the relationship between Asian Americans’ English proficiency and suicide ideation.

Although the foregoing literature review supports the idea that indicators of family relationships and acculturation might be factors associated with Asian Americans' suicidal behavior, there has been a lack of research on the interaction between both factors. In the only known study on this topic, Lau et al. (2002) found that family conflict was more strongly related to suicidal behavior among less acculturated Asian American youth than among highly acculturated Asian American youth. Arguably, a broadly similar outcome might apply to Asian American adults. Asian American adults with low levels of English proficiency might be more reliant on their family members to navigate the demands of the dominant culture; therefore, a breakdown in family cohesion might be especially stressful for them.

\section{Psychological Distress as a Mediator}

In addition to examining potential interaction effects involving family cohesion and English proficiency, we also sought to investigate a potential mediator of the relationship between Asian Americans' family cohesion and suicide ideation. The identification of mediators has important clinical implications: in situations where the enhancement of family cohesion is not a realistic therapeutic goal, clinicians can intervene to reduce the impact of mediating risk factors to prevent the development of suicidal behaviors among Asian Americans. 
In the present study, we examined psychological distress as a potential mediator of the link between family cohesion and suicide ideation. Indicators of psychological distress have been implicated as predictors of suicidal ideation and behavior in the general U.S. population (Kessler, Berglund, Borges, Nock, \& Wang, 2005; Westefeld et al., 2000) as well as among Asian Americans (Aruna, 2001; Lau et al., 2002; Yang \& Clum, 1994). Further, family conflict has been shown to be related to psychological distress among Asian Americans (Lee, Jung, Su, Tran, \& Bahrassa, 2009). Collectively, these findings converge on the notion that a lack of family cohesion may be a risk factor for psychological distress, which, in turn, engenders suicide ideation among Asian Americans.

\section{Hypotheses}

We proposed four hypotheses in this study of suicide ideation in a national, adult community sample of Asian Americans. First, we hypothesized that family cohesion would be negatively related to suicide ideation. Second, we anticipated that English proficiency would be negatively related to suicide ideation. Third, we expected that English proficiency would moderate the association between family cohesion and suicide ideation; that is, this association would be stronger among Asian Americans with low levels of English proficiency than among those with high levels of English proficiency. Fourth, we predicted that psychological distress would mediate the relationship between family cohesion and suicide ideation.

\section{Method}

\section{Participants and Procedures}

This study involved an archival analysis of data drawn from the National Latino and Asian American Study (NLAAS), the first national epidemiological study of Asian Americans' and Latinos' mental health. For this study, only the Asian American sample was used. The 
original sample consisted of 2095 non-institutionalized Asian Americans representative of the national population of Asian American adults across the 50 states of the U.S., although Chinese, Filipino, and Vietnamese Americans were oversampled.

The deletion of cases $(1.1 \% ; N=23)$ with missing data on the main variables resulted in a final sample of 2072 participants (47.7\% female, 52.3\% male). Participants' average age was 41.20 years $(S D=14.76$, range $=18-95)$. Participant identified as Chinese $(28.4 \%)$, Filipino (24.4\%), Vietnamese (24.8\%), and other Asian ethnicities (e.g., Japanese, Korean, Indian, etc.; 22.4\%). $78.2 \%$ of participants were born outside the United States, a percentage that is higher than that of the U.S. Asian population (69\%; U.S. Census Bureau, 2000). Immigrant participants had lived in the United States for an average of 13.74 years $(\mathrm{SD}=9.76)$. Participants' highest education level attained included less than 12 years $(15.0 \%), 12$ years $(17.5 \%), 13-15$ years $(25.4 \%)$, and 16 and more years $(42.2 \%)$. The proportion of college graduates in our sample is slightly lower than that of the U.S. Asian and Pacific Islander population 25 years and above (47\%; U.S. Census Bureau, 2002).

The data for NLAAS was collected between May 2002 and November 2003 using a multistage, stratified area probability sampling design. The recruitment procedure involved three components: (a) core sampling based on a nationally representative sample of Asian Americans regardless of residential patterns, (b) high density sampling in areas with a $5 \%$ or more density of Asian American households, and (c) the sampling of second respondents from households in which a primary respondent had already been interviewed. A survey was administered face-toface in participants' homes by trained interviewers with linguistically and culturally similar backgrounds unless participants requested telephone interviews (see Pennell et al., 2004, for further details on recruitment procedures and the training of interviewers). All items in the 
survey were translated from English to Asian languages using back-translation procedures. $64.3 \%(N=1333)$ of participants completed the interviews in English. For the purposes of this study, only the following measures relevant to our study aims were used for analyses.

\section{Instruments}

Suicide Ideation. The World Mental Health Survey Initiative version of the World Health Organization-Composite International Diagnostic Interview is a cross-cultural psychiatric structured diagnostic interview designed to be administered by trained lay interviewers (WMH-CIDI; Kessler \& Ustun, 2004). Suicide ideation was measured using one item in the WMH-CIDI. Participants were asked "Have you ever seriously thought about committing suicide?" and if so, they were asked, "Have you seriously thought about committing suicide at any time in the past 12 months?" Participants' responses were categorized as 1 (if they endorsed suicide ideation in the past 12 months) and 0 (if they did not). Evidence for the validity of the WMH-CIDI has been shown in diverse samples across 28 countries, including several Asian countries (Kessler \& Ustun). The suicide ideation item in the WMH-CIDI was found to be significantly related to anxiety and mood disorders in a sample of Chinese across several metropolitan areas in China (Lee et al., 2007).

Psychological Distress. Psychological distress was measured by the 6-item Kessler Psychological Distress Scale (K6; Kessler et al., 2002). K6 is a measure of non-specific psychological distress used in annual government health surveys in the U.S. and Canada. Participants were asked about often during the last 30 days they experienced a series of symptoms on a 4-point scale. Response options range from none of the time to all of time. A sample item is "how often did you feel worthless?" K6 does not include items related to suicide ideation. The overall score was determined by averaging participants' responses to all items. 
Kessler et al. (2002) reported evidence of strong psychometric properties across diverse sociodemographic groups, including K6's ability to discriminate between community cases and non-cases of DSM-IV disorders. Evidence for K6's construct validity in a community sample of Asian Americans was demonstrated through associations with chronic illness, less education, unemployment, low levels of family income, and being unmarried (Bratter \& Eschbach, 2005). For our sample, the internal consistency of K6 was .78.

Family Cohesion. Family cohesion was measured using the 10-item Cohesion subscale of the Family Adaptation and Evaluation Scale (FACES III; Olson, 1986). Participants were asked the extent to which they agreed or disagreed with statements about their family (no definition of “family" was provided). Response options range from 1 to 4 . Examples of items are "family members like to spend free time with each other" and "Family togetherness is very important." High scores represent greater family cohesion. Preliminary evidence for the construct validity of the Cohesion subscale for Asian Americans was demonstrated through correlations with ethnic identity and psychological distress in another NLAAS study based largely on the same sample as that used in our study (Yip, Gee, \& Takeuchi, 2008). For our sample, the internal consistency of the Cohesion subscale scores was .93 .

English Proficiency. Participants' English proficiency was measured using the 3-item English Proficiency Scale of the Cultural Identity Scales for Latino Adolescents (Felix-Ortiz, Newcomb, \& Myers, 1994). Participants were asked, “How well do you speak/read/write English?” Response options were poor (1), fair (2), good (3), and excellent (4). Total scores were divided by 3 , providing a possible range of scores from 1 to 4 . Our sample was moderately diverse in terms of English proficiency $(M=2.80, \mathrm{SD}=1.02$, range $=1-4)$; although the majority of participants $(58.8 \%)$ had scores in the good to excellent range $(3-4), 41.2 \%$ and $18.8 \%$ 
indicated that their English proficiency was less than good (3) and less than fair respectively (2). No previous validity studies using the English Proficiency Scale with Asian Americans had been conducted. In our sample, preliminary evidence of construct validity was demonstrated through a negative association with being an immigrant $(r=-.43, p<.001)$ and a positive association with level of education $\left(r_{s}=.44, p<.001\right)$. Cronbach's alpha for our sample was .97 .

\section{Results}

\section{Preliminary Analyses}

A series of tests were used to examine potential influences of demographic variables on suicide ideation. Chi-square tests were used to analyze the relationships between suicide ideation and sex, place of birth (U.S. versus outside the U.S.), type of Asian ethnicity, and level of education; a $t$-test was used to examine age differences between suicide ideators and nonideators. The Bonferroni-corrected significance level was set at $p<.01$ for all five tests. The relationships between suicide ideation and sex, Asian ethnicity, and education were not significant. However, place of birth was significantly associated with suicide ideation, $\chi^{2}(1)=$ 17.34, $p<.001$. U.S.-born participants were more likely to have reported having suicide ideation. In addition, suicidal participants tended to be younger $(M=31.18$ years; $S D=7.40)$ than nonsuicidal participants $(M=41.37$ years; $S D=14.79), t(36.28)=7.67, p<.001$. Consequently, age and place of birth were included as covariates in the subsequent main analyses. Means, standard deviations, and interrcorrelations among the main variables and covariates are presented in Table 1. In addition, $1.6 \%$ of participants $(N=33)$ reported suicide ideation within the past 12 months.

\section{Main Analyses}

We used hierarchical logistic regression to examine predictors of suicide ideation (ideation versus no ideation) in our sample of 2072 participants. Logistic regression is the 
appropriate statistical method for analyses involving dichotomous outcomes variables. Unlike multiple regression, logistic regression does not assume that the outcome variable is normally distributed and that there is a linear relationship between the predictor and outcome variables (Cizek \& Fitzgerald, 1999). Age and place of birth ( $0=$ U.S.-born; $1=$ born outside the U.S.), were included as control variables in the first step. In the second step, family cohesion and English proficiency were added. Finally, in the third step, the interaction between family cohesion and English proficiency was included. Scores were standardized to reduce multicollinearity. Table 2 summarizes the results of this analysis.

The omnibus tests of model coefficients showed that the full model was significant, $\chi^{2}=$ $56.70, p<.001$. The Nagelkerke $R^{2}$ indicated that $18 \%$ of the variance in suicide ideation was accounted for by the predictor variables collectively. Place of birth and English proficiency were not significantly associated with suicide ideation. In contrast, age, family cohesion, and the family cohesion x English proficiency interaction were significant predictors of suicide ideation. Specifically, younger Asian Americans as well as Asian Americans with lower levels of family cohesion had increased odds of having suicide ideation.

To interpret the significant family cohesion $\mathrm{x}$ English proficiency interaction effect, the odds ratio for family cohesion was examined at one standard deviation above and below the mean of English proficiency scores. Family cohesion was more strongly related to a lower odds of suicide ideation among low English proficiency participants $(\mathrm{OR}=.30,95 \% \mathrm{CI}=.18, .51, p<$ $.05)$ than among high English proficiency participants $(\mathrm{OR}=.67,95 \% \mathrm{CI}=.52, .86, p<.05)$. We also interpreted these findings in terms of the odds of having suicide ideation (i.e., 1 divided by OR): high English proficiency participants were 1.49 times more likely to have suicide ideation when family cohesion was low; in contrast, low English proficiency participants were 3.33 times 
more likely to have suicide ideation when family cohesion was low. In sum, our moderation hypothesis was supported.

To test our mediation hypothesis, we used a bootstrapping procedure, which involved repeatedly sampling from the original data to provide an empirical approximation of the sampling distribution of the indirect effect (Preacher \& Hayes, 2008). Unlike the commonly-used procedure for testing mediation proposed by Baron and Kenny (1986), the bootstrapping procedure can accommodate dichotomous outcome variables and does not assume that the sampling distribution of the indirect effect is normal. Using an SPSS macro (Hayes, 2009) associated with the bootstrapping procedure described in Preacher and Hayes, 10,000 bootstrap samples were created by randomly sampling with replacements from the original data, yielding 10,000 estimates of the indirect path coefficients (covariates were age, place of birth, and English proficiency). The mean estimate of the indirect effect was -.06, SE $=.01$, bias-corrected $95 \% \mathrm{CI}=-.079,-.040$. Because the confidence intervals did not include zero, the hypothesized mediated effect from family cohesion to suicide ideation through psychological distress was significant at $p<.05$. A posthoc logistic regression was conducted with place of birth, age, English proficiency, family cohesion, and psychological distress as the predictor variables and suicide ideation as the outcome variable. Controlling for all other variables, psychological distress was significantly associated with suicide ideation, $B=1.48, \mathrm{SE}=.20, \mathrm{OR}=4.40,95 \%$ $\mathrm{CI}=2.95,6.56, p<.001$. Family cohesion was significantly and negatively related to suicide ideation after controlling for all other variables $(B=-.06, \mathrm{SE}=.03, \mathrm{OR}=.94,95 \% \mathrm{CI}=.90, .99$, $p=.029)$, suggesting that the mediation effect was partial rather than complete. Place of birth and English proficiency were not significantly related to suicide ideation, $p>.05$. However, age 
remained significantly and negatively related to suicide ideation after controlling for all other variables, $B=-.06, \mathrm{SE}=.02, \mathrm{OR}=.94,95 \% \mathrm{CI}=.91, .98, p=.002$.

Because causality cannot be assumed from cross-sectional data, we tested a theoretically plausible alternative model with family cohesion as a mediator between psychological distress and suicide ideation. We repeated the above bootstrapping procedure. The mediation effect for the alternative model was not significant at $p<.05$ because the confidence intervals included zero (mean estimate of the indirect effect $=.10, \mathrm{SE}=.05$, bias-corrected $95 \% \mathrm{CI}=-.005, .194)$. Hence, the hypothesized model with psychological distress as a mediator between family cohesion and suicide ideation was a better fit with the data than the alternative model.

\section{Discussion}

The purpose of this study was to apply the construct of failed belongingness in Joiner's (2005) interpersonal theory to the study of family cohesion and suicide ideation in a national, adult community sample of Asian Americans. Our first hypothesis that family cohesion would be negatively related to suicide ideation was supported. A lack of family cohesion may contribute to an increased risk for the onset of suicidal behavior (Lau et al., 2002; Leong et al., 2007). Conversely, family cohesion may be a protective factor against suicidal behavior among Asian Americans. This result is consistent with scholarly assertions that in Asian cultures, the family is viewed as individuals' primary source of support and security (Inman \& Yeh, 2007; Tsoh et al., 2005).

Our second hypothesis that English proficiency would be negatively related to suicide ideation was not supported. However, age was negatively associated with suicide ideation. The elevated levels of suicide ideation among younger Asian American adults dovetail with research showing that Asian American college students reported higher levels of suicide ideation than 
their European American counterparts (Brener, Hassan, \& Barrios, 1999). Perhaps the developmental challenges related to being in college, beginning their careers, or starting new families are particular stressful for younger Asian American adults because they face competing demands of navigating the cultural expectations of their parents and those of their peers, colleagues, or new families. Another possibility is that the relationship between age and suicide ideation reflects reluctance among older Asian Americans to disclose suicide ideation because of perceived stigma attached to such disclosure. These suggestions are, at best, speculatory and are subject to future empirical research.

Consistent with our third hypothesis, English proficiency moderated the association between family cohesion and suicide ideation. The negative relationship between family cohesion and suicide ideation was stronger among low English proficiency Asian Americans than among high English proficiency Asian Americans. In other words, a lack of family cohesion appeared to be a greater suicide ideation risk factor for low English proficiency participants as compared with high English proficiency participants. Asian Americans who are less proficient in English may be more reliant on their families to negotiate the demands of the dominant culture. For example, a first-generation, widowed Vietnamese American woman who cannot speak or read English might have to rely on her children to assist with activities such as paying her utility bills and visiting a doctor. Consequently, a lack of family cohesion is likely to have more deleterious consequences for Asian Americans with low levels of English proficiency.

Our fourth hypothesis on the mediating role of psychological distress received support. Our findings are consistent with a model in which a lack of family cohesion was related to psychological distress, which was in turn, associated with suicide ideation among Asian Americans. Nonetheless, the mediating role of psychological distress was partial rather than 
complete, demonstrating that a lack of family cohesion remained a relevant suicide ideation risk factor that cannot be completely accounted for by its association with psychological distress.

An important strength of this study is the use of a large, national community sample of Asian American adults that is diverse in age, English proficiency, ethnicity, and education. A limitation of previous research on Asian Americans' suicide ideation is that the majority of studies have relied on college samples (e.g., Jha, 2001;Yang \& Clum, 1994). Whereas an Asian American college sample is likely to have a restricted range of age and English proficiency, the variability in age and English proficiency in our sample may have enabled the detection of the significant relationship between age and suicide ideation as well as the significant family cohesion by English proficiency interaction effect.

\section{Limitations and Directions for Future Research}

There were several limitations in our study. First, because of the cross-sectional nature of the study, causality among the variables cannot be assumed. We recommend the future use of longitudinal data to clarify the causal direction of the relationships among Asian Americans' family cohesion, English proficiency, psychological distress, and suicide ideation.

Second, this study only examined one cultural variable - English proficiency. B. S. K. Kim (2007) has argued that the process through which Asian Americans adhere to cultural norms should be conceptualized as bilinear (acculturation to European American culture and enculturation to one's Asian culture) and multidimensional (including a behavioral dimension and a values dimension). English proficiency merely constitutes one aspect of behavioral acculturation. Future studies on Asian Americans' suicidal behavior should examine other cultural constructs (e.g., the values dimension of acculturation and enculturation). For instance, researchers can investigate whether the link between Asian Americans' unmet interpersonal 
needs and suicidal behavior is moderated by adherence to traditional Asian values, such as selfsacrifice and social obligations (Author \& ZZ, in press).

Third, our findings must be interpreted cautiously in light of limitations in our measures. Because the definition of "family" was not provided in the Cohesion subscale of FACES III (Olson, 1986), it was unclear whether participants responded based on their family of origin, nuclear family, or extended family and whether their understanding of "family" differed as a function of their age. Therefore, in interpreting the significant association between family cohesion and suicide ideation, we cannot pinpoint the specific types of familial bonds that buffer against suicide ideation. In addition, our use of a 1-item dichotomous measure of suicide ideation (i.e., whether participants seriously thought of death by suicide or not) might have failed to detect the presence of mild suicidal thoughts (e.g., a vague desire for death). Future studies should use measures that can detect a broader range of suicidal thoughts. Nonetheless, it should be noted that the use of a 1-item dichotomous measure of suicide ideation is a common practice in large scale epidemiological studies, such as the National Comorbidity Survey (Kessler et al., 2005) and the National College Health Risk Behavior Survey (Brener et al., 1999); importantly, both of these surveys used a suicide ideation item that was similar in wording to that used in our study.

\section{Practical Implications}

Our findings present several practical implications. Mental health professionals can provide preventative psychoeducation to Asian American families on the potential link between family cohesion and suicide ideation as well as strategies for strengthening family cohesion. With regard to counseling practice, the assessment of culture variables should be integral to any work with suicidal Asian Americans (Choi et al., 2009). Our findings indicate that Asian 
Americans who experience a combination of low English proficiency and a lack of family cohesion may be particularly vulnerable to developing suicide ideation. Recommendations for strengthening familial bonds among such Asian Americans include involving family members in the therapeutic process and encouraging family members to describe ways in which clients contribute to their families' welfare (Author \& ZZ, in press). Moreover, scholars have observed that intergenerational cultural conflicts between parents and children may be a common phenomenon in Asian American families (Choi et al., 2009). For example, some U.S.-born Asian Americans might find their cultural values incongruent with those of their immigrant parents as a result of acculturating more rapidly to the dominant culture. Mental health professionals can help strengthen family cohesion in Asian American families by finding ways to bridge this cultural gap in family therapy.

Finally, because mental health professionals are likely to work with suicidal clients at some point in their careers, Westefeld et al. (2000) recommended that training in suicide assessment and intervention should be an important feature of the training of mental health professionals. In view of our study's findings as well as recent scholarship on the intersection of culture and suicidality (e.g., Choi et al., 2009; Leong et al., 2007), we propose that the influence of cultural variables on suicidal behavior should be a core component of such training. 


\section{References}

American Association of Suicidology (2008). U.S.A. suicide: 2008 official final data. Retrieved December 4, 2008, from http://www.suicidology.org/associations/1045/files/2005datapgs.pdf.

Author, XX, \& YY. (2009) Risk and protective factors associated with Asian American students' suicide ideation: A multi-campus, national study. Manuscript submitted for publication.

Author, \& ZZ. (in press). Counseling Asian American men with suicidal behavior. In W. M. Liu, D. Iwamoto, \& M. Chae (Eds.), Culturally responsive counseling with Asian American men. New York: Routledge.

Baron, R. M., \& Kenny, D. A. (1986). The moderator-mediator variable distinction in social psychological research: Conceptual, strategic, and statistical considerations. Journal of Personality and Social Psychology, 51, 1173-1182.

Bratter, J. L., \& Eschbach, K. (2005). Race/ethnic differences in non-specific psychological distress: Evidence from the National Health Interview Survey. Social Science Quarterly, 86, 620-644.

Brener, N. D., Hassan, S. S., \& Barrios, L. C. (1999). Suicidal ideation among college students in the United States. Journal of Consulting and Clinical Psychology, 67, 1004-1008.

Choi, J. J., Rogers, J. R., \& Werth, J. L. (2009). Suicide risk assessment with Asian American college students: A culturally informed perspective. The Counseling Psychologist, 37, $186-218$

Cizek, G. J., \& Fitzgerald, S. M. (1999). An introduction to logistic regression. Measurement and Evaluation in Counseling and Development, 31, 223-245. 
Felix-Ortiz, M., Newcomb, M. D., \& Myers, H. (1994). A multidimensional measure of cultural identity for Latino and Latina adolescents. Hispanic Journal of Behavioral Sciences, 16, 99-115.

Hayes, A. (2009, March). SPSS macro to estimate total indirect and specific indirect effects and bootstrap confidence intervals in single-step mediator models.[Computer software]. Retrieved June 13, 2009, from http://www.comm.ohio-state.edu/ahayes.

Hwang, W., Chun, C., Takeuchi, D. T., Myers, H. F., \& Siddart, P. (2005). Age of first onset major depression in Chinese Americans. Cultural Diversity and Ethnic Minority Psychology, 11, 16-27.

Inman, A. G., \& Yeh, C. (2007). Asian American Stress and Coping. In F. T. L. Leong, A. Ebreo, L. Kinoshita, A. G. Inman, L. H. Yang, L. Kinoshita, et al. (Eds.), Handbook of Asian American Psychology (2nd ed., pp. 323-339). Thousand Oaks, CA: Sage Publications, Inc.

Jeong, G. J., \& Schumm, W. R. (1990). Family satisfaction in Korean/American marriages: An exploratory study of the perceptions of Korean wives. Journal of Comparative Family Studies, 21, 325-335

Joiner, T. (2005). Why people die by suicide. Cambridge, MA: Harvard University Press.

Joiner, T. E., Jr., Van Orden, K. A., Witte, T. K., \& Rudd, M. D. (2009). The interpersonal theory of suicide: Guidance for working with suicidal clients. Washington, DC: American Psychological Association.

Jha, A. (2001). Depression and suicidality in Asian Indian students. Dissertation Abstracts International, 62(03), 1311B. 
Kang, S. M. (2006). Measurement of acculturation, scale formats, and language competence: Their implications for adjustment. Journal of Cross-Cultural Psychology, 37, 669-693.

Kessler R. C., Andrews G., Colpe L. J., Hiripi E., Mroczek, D. K., Normand S. L., et al. (2002). Short screening scales to monitor population prevalences and trends in non-specific psychological distress. Psychological Medicine, 32, 959-76.

Kessler, R. C., Berglund, P., Borges, G., Nock, M., \& Wang, P. S. (2005). Trends in suicide ideation, plans, gestures, and attempts in the United States, 1990-1992 to 2001-2003. Journal of the American Medical Association, 293, 2487-2495.

Kessler, R. C., \& Ustun, B. (2004). The World Mental Health (WMH) Survey Initiative version of the World Health Organization (WHO) Composite International Diagnostic Interview (CIDI). International Journal of Methods in Psychiatric Research, 13, 93-121.

Kim, B. S. K. (2007). Acculturation and enculturation. In F. T. L. Leong, A. Ebreo, L. Kinoshita, A. G. Inman, L. H. Yang, L. Kinoshita, et al. (Eds.), Handbook of Asian American Psychology (2nd ed., pp. 141-158). Thousand Oaks, CA: Sage.

Kim, B. S. K., Atkinson, D. R., \& Umemoto, D. (2001) Asian cultural values and the counseling process: Current knowledge and directions for future research. The Counseling Psychologist, 29, 570-603.

Kung, H. C., Hoyert, D. L., Xu, J., \& Murphy, S.L. (2008). Deaths: Final data for 2005. National Vital Statistics Report, 56, 1-66.

Lam, R.E., Pacala, R.E. \& Smith, S.L. (1997). Factors related to depressive symptoms in an elderly Chinese-American sample. Clinical Gerontologist, 17, 57-70. 
Lau, A. S., Jernewall, N. M., Zane, N., \& Myers, H. F. (2002). Correlates of suicidal behaviors among Asian American outpatient youths. Cultural Diversity and Ethnic Minority Psychology, 8, 199-213.

Lee, R.M., Jung, K.R., Su, J.C., Tran, A.G.T.T., \& Bahrassa, N. (2009). The family life and adjustment of Hmong American sons and daughters. Sex Roles, 60, 549-558.

Leong, F. T. L., Leach, M. M., Yeh, C., \& Chou, E. (2007). Suicide among Asian Americans: What do we know? What do we need to know? Death Studies, 31, 417-434.

Mann, J. J. (2002). A current perspective of suicide and attempted suicide. Annals of Internal Medicine, 136, 302-311.

Markus, H. \& Kitayama, S. (1991). Culture and the self: Implications for cognition, emotion, and motivation. Psychological Review, 98, 224-253.

Maris, R. W., Berman, A. L., \& Silverman, M. M. (2000). Comprehensive textbook of suicidology. New York, NY: Guilford Press.

Olson, D. H. (1986). Circumplex Model VII: Validation studies and FACES III, Family Process, $25,337-351$.

Pennell, B., Bowers, A., Carr, D., Chardoul, S., Cheun, G., Dinkelmann, K.. et al. (2004). The development and implementation of the National Comorbidity Survey Replication, the National Survey of American Life, and the National Latino and Asian American Survey. International Journal of Methods in Psychiatric Research, 13, 241-269.

Preacher, K. J., \& Hayes, A. F. (2008). Asymptotic and resampling strategies for assessing and comparing indirect effects in multiple mediator models. Behavior Research Methods, 40, $879-891$.

Range, L. M., Leach, M. M., McIntyre, D., Posey-Deters, P. B., Marion, M. S., Kovac, S. H., et al. (1999). Multicultural perspectives on suicide. Aggression \& Violent Behavior, 4, 413-430. 
Shiang, J., Blinn, R., Bongar, B., Stephens, B., Allison, D., \& Schatzberg, A. (1997). Suicide in San Francisco, CA: A comparison of Caucasian and Asian groups, 1987-1994. Suicide \& Life-Threatening Behavior, 27, 80-91.

Tsoh, J., Helen, F. K., Duberstein, P. R., Chan, S. S. M., Chi, I., Yip, P. S. F., et al. (2005). Attempted suicide in elderly Chinese persons: A multi-group, controlled study. American Journal of Geriatric Psychiatry, 13, 562-571.

U.S. Census Bureau (2000). The Foreign-Born Population: 2000. Retrieved December 19, 2008 from http://www.census.gov/prod/2003pubs/c2kbr-34.pdf.

U.S. Census Bureau (2002). Current Population Survey. Retrieved June 17, 2009 from http://www.census.gov/Press-Release/www/releases/archives/education/000818.html.

U. S. Department of Health and Human Services (2001). National strategy for suicide prevention: Goals and objectives for action. Retrieved December 5, 2008 from http://download.ncadi.samhsa.gov/ken/pdf/SMA01-3517/SMA01-3517.pdf.

Westefeld, J. S., Range, L. M., Rogers, J. R., Maples, M. R., Bromley, J. L., \& Alcorn, J. (2000). Suicide: An overview. The Counseling Psychologist, 28, 445-510.

Yang, B., \& Clum, G. A. (1994). Life stress, social support, and problem-solving skills predictive of depressive symptoms, hopelessness, and suicide ideation in an Asian student population: A test of a model. Suicide \& Life-Threatening Behavior, 24, 127-139.

Yip, T., Gee, G. C., \& Takeuchi, D. T. (2008). Racial discrimination and psychological distress: The impact of ethnic identity and age among immigrant and United States-born Asian adults. Developmental Psychology, 44, 787-800. 
Table 1

Intercorrelations, Means, and Standard Deviations for the Main Variables $(N=2072)$

\begin{tabular}{|c|c|c|c|c|c|c|c|c|c|}
\hline & Variables & $\mathrm{M}$ & SD & 1. & 2. & 3. & 4. & 5. & 6. \\
\hline 1. & Age & 41.20 & 14.76 & - & $.16^{*}$ & $.14^{*}$ & $-.34 *$ & -.01 & $-.09 *$ \\
\hline 2. & Place of Birth & - & - & & - & $.17^{*}$ & $-.43^{*}$ & .01 & $-.09 *$ \\
\hline 3. & Family Cohesion & 36.85 & 4.62 & & & - & $-.12 *$ & $-.25 *$ & $-.16^{*}$ \\
\hline 4. & $\begin{array}{l}\text { English } \\
\text { Proficiency }\end{array}$ & 2.80 & 1.03 & & & & - & -.04 & $.07 *$ \\
\hline 5. & $\begin{array}{l}\text { Psychological } \\
\text { Distress }\end{array}$ & 1.50 & .69 & & & & & - & $.23 *$ \\
\hline 6. & Suicide Ideation & - & - & & & & & & - \\
\hline
\end{tabular}


Table 2 .

Predictors of Suicide Ideation Using Hierarchical Logistic Regression $(N=2072)$

\begin{tabular}{|c|c|c|c|c|c|c|}
\hline \multirow[t]{2}{*}{ Step } & \multirow[t]{2}{*}{ Variable } & \multirow[t]{2}{*}{$B$} & \multirow[t]{2}{*}{$S E$} & \multirow{2}{*}{$\begin{array}{l}\text { Odds } \\
\text { Ratio }\end{array}$} & \multicolumn{2}{|c|}{$95 \% \mathrm{CI}$} \\
\hline & & & & & Lower & Upper \\
\hline \multirow[t]{2}{*}{ Step 1} & Place of Birth & -1.06 & .37 & $.35 *$ & .17 & .71 \\
\hline & Age & -.76 & .24 & $.47 *$ & .29 & .75 \\
\hline \multirow[t]{4}{*}{ Step 2} & Place of Birth & -.78 & .42 & .46 & .20 & 1.04 \\
\hline & Age & -.67 & .25 & $.51 *$ & .31 & .84 \\
\hline & Family Cohesion & -.56 & .11 & $.57 * *$ & .46 & .71 \\
\hline & English Proficiency & .06 & .26 & 1.06 & .64 & 1.75 \\
\hline \multirow[t]{5}{*}{ Step 3} & Place of Birth & -.75 & .41 & .47 & .21 & 1.06 \\
\hline & Age & -.70 & .26 & $.50 *$ & .30 & .82 \\
\hline & Family Cohesion & -.80 & .14 & $.45^{* *}$ & .34 & .59 \\
\hline & English Proficiency & .50 & .34 & 1.64 & .84 & 3.19 \\
\hline & $\begin{array}{l}\text { Family Cohesion } x \\
\text { English Proficiency }\end{array}$ & .39 & .15 & $1.48 *$ & 1.11 & 1.98 \\
\hline
\end{tabular}

